\title{
Study of Combining DVB-SH Signals from Satellite and Terrestrial Repeater
}

\author{
Hui Zhou
}

\begin{abstract}
In DVB-SH (Digital Video Broadcasting-Satellite services to Handhelds) systems, mobile terminals can receive either a signal from a broadcasting satellite or a signal from a terrestrial repeater. These two signals are not combined at the mobile receiver. The technique of signal combining is widely used in terrestrial mobile communication systems, and is effective against fading and multipath effects to improve the quality of received signals. Thus, the purpose of this paper is to discuss the feasibility of signal combining for DVB-SH terminals and to find out whether there is an improvement to be obtained by combining the signals from satellite and terrestrial repeater. In the paper, we first perform a statistical analysis of channels in different states. Then, we discuss two methods for combining the two signals. Finally, we analyze the improvement of each method.
\end{abstract}

Index Terms-DVB-SH, spatial diversity, LMS, fading, multipath.

\section{INTRODUCTION}

DVB-SH is the name of a mobile broadcast standard designed to deliver video, audio and data services to small handheld devices such as mobile telephones, and to vehicle-mounted devices. The key feature of DVB-SH is the fact that it is a hybrid satellite/terrestrial system that will allow the use of a satellite to achieve coverage of large regions or even a whole country. In areas where direct reception of the satellite signal is impaired, and for indoor reception, terrestrial repeaters are used to improve service availability [1].

It means that, in DVB-SH systems, mobile terminals can received either a signal from a broadcasting satellite or a signal from a terrestrial repeater. These two signals are not combined at the mobile receiver.

In the first case, mobile terminals only receive signal from satellite. The quality of signal received by mobile terminal depends on shadowing and multipath fading in LMS channel. If there is LOS (Line of Sight) signal with little multipath effect, the performance is considerable. However, when the multipath fading becomes severe, especially when there is no LOS signal, the performance is coming down sharply.

In the second case, mobile terminals only receive signal from repeater. The quality of signal received by mobile terminal depends on shadowing and multipath fading in terrestrial channel which is worse than in LMS channel. The reason is that the elevation angle from the repeater to mobile terminal is much less than that from the satellite. So that there

Manuscript received December 29, 2013; revised February 28, 2014.

Hui Zhou is with the Institute of Telecommunication Satellite, CAST, Beijing, China, 100094 (e-mail:cast_zhouhui@163.com). is no LOS signal in most of time. Furthermore, the shadowing fading and multipath effect is more severe than in LMS (Land Mobile Satellite) channel.

As a result, it is necessary to find a way to solve these problems. In terrestrial mobile communication systems, an effective way to deal with fading and multipath effect is spatial diversity. The technique can also be utilized in our case. The purpose is to discuss the feasibility of signal combining for DVB-SH terminals and to find out whether there is an improvement to be obtained by combining the signals from satellite and terrestrial repeater. In this paper, we first perform a statistical analysis of channels according to good and bad states. Then, we discuss two methods for combining the signals from satellite and terrestrial repeater. Finally, we analyze the improvement of each method based on simulated results.

\section{StatistiCAl ANALYSIS OF CHANNELS}

In order to make models and get results for combination, it necessary to make channel analysis. The LMS channel is analyzed based on statistics. As is known, there are several LMS models available for statistical analysis [2]-[5]. Lutz model is a widely used two state model for LMS channel analysis, as a result, the statistical analysis in this chapter is based on Lutz model and measured result.

According to Lutz model, the LMS channel is distinguished into two states: one is good state, the other one is bad state. The good channel state corresponds to areas without an obstructed view of the satellite (un-shadowed areas), whereas the bad channel state represents areas where the direct satellite signal is shadowed by obstacles. In both of these cases, the satellite signal is reflected from a large number of objects in the surroundings of the mobile receiver, which leads to multipath signal [3].

\section{A. Good State}

In good state, there is no shadowing, thus the multipath signal is combined with the LOS signal from satellite. The total received signal is Rician fading in this case, which means that the amplitude of the signal is Rice distributed random process. Thus the momentary received power $S$ obeys a Rician probability density (with the mean received power of LOS signal normalized to unity):

$$
p_{\text {rice }}(S)=c e^{-c(S+1)} I_{0}(2 c \sqrt{S})
$$

where $S$ is the received power, $c$ is the Rice factor which is equal to the direct-to-multipath signal power ratio, $I_{0}$ is the 
modified Bessel function of order zero which is defined as follows.

$$
I_{0}(x)=1+\frac{(x / 2)^{2}}{(1 !)^{2}}+\frac{(x / 2)^{4}}{(2 !)^{2}}+\frac{(x / 2)^{6}}{(3 !)^{2}}+\cdots
$$

As a result, the effect of the multipath fading depends on the Rice factor. Different area has different Rice factor. The Rice factor equal to $5.5 \mathrm{~dB}$ in city environment with elevation of $43^{\circ}$ according to measured results [3] is used for the rest analysis.

\section{B. Bad State}

In this case, the shadowing is present which means that direct signal path no longer exists. The received signal is affected from both shadowing and multipath fading. The effect of the shadowing leads to a very slow variance of fading, while the multipath leads to fast variance of fading.

The shadowing process results in a time varying short-term mean received signal power (relative to LOS power). The probability of the signal power has the characteristics of lognormal distribution. The PDF is defined as follows.

$$
p_{L N}\left(S_{0}\right)=\frac{10}{\sqrt{2 \pi} \sigma \ln 10} \cdot \frac{1}{S_{0}} \exp \left[-\frac{\left(10 \log S_{0}-\mu\right)^{2}}{2 \sigma^{2}}\right] \text { (3) }
$$

where $S_{0}$ is the short-term mean received power, $\mu$ is the mean power level decrease $(\mathrm{dB}), \sigma^{2}$ is the variance of the power level due to shadowing.

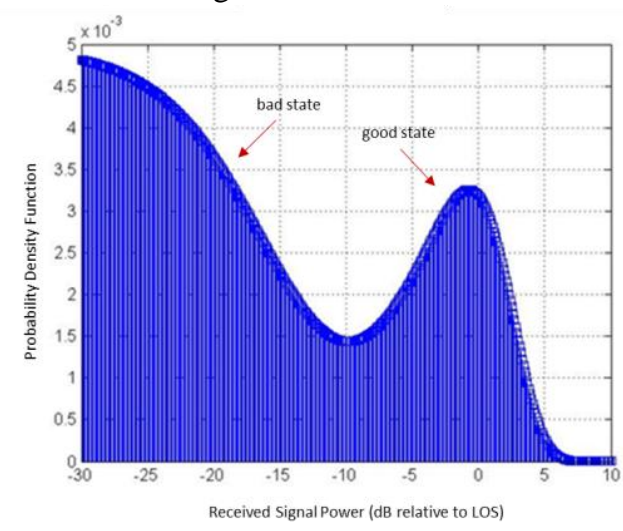

Fig. 1. Probability density function of received signal in LMS channel.

As a result, the effect of the shadowing fading depends on two factors: mean power level decrease $(\mu)$ and variance of power level $(\sigma)$. The factors depend on the area. The mean power level decrease is equal to $-13.6 \mathrm{~dB}$ and variance of power level is equal to $3.8 \mathrm{~dB}$ according to measured results [3].

As for the multipath fading in bad state, the probability density function of the received power conditioned on the short-term mean power $S_{0}$ is as follows.

$$
P_{\text {Ray } 1}\left(S \mid S_{0}\right)=\frac{1}{S_{0}} \exp \left(-\frac{S}{S_{0}}\right)
$$

When choosing $S_{0}$ as the minimum power related to $90 \%$ probability, the probability density function of received power in bad channel state and good state can be obtained respectively.

\section{Total Probability}

In order to get the total probability of received signal power, the percentage of shadowing $A$ is defined. So the resulting probability density function becomes:

$$
P(S)=(1-A) P_{\text {Rice }}(S)+A \int_{0}^{\infty} R_{\text {Ray } 1}\left(S \mid S_{0}\right) P_{L N}\left(S_{0}\right) d S_{0}
$$

The percentage of shadowing is equal to $54 \%$ according to measured results, so that the total probability density function of received signal can be shown in Fig. 1.

It shows that the maximum probability of received signal power in good state is around $-1 \mathrm{~dB}$ relative to LOS signal power and the variance is about $\pm 5 \mathrm{~dB}$, whereas the received power in bad state has the range below $-10 \mathrm{~dB}$.

For terrestrial channel, the shadowing fading is also severe that direct signal path no longer exist in most of time, thus the probability density of received signal is assumed the same as bad state in LMS channel for the rest analysis.

\section{Methods AND Results OF COMBINATION}

\section{A. Methods of Combination}

According to DVB-SH standard, there are two scenarios for the mobile terminals:

1) The signal from the satellite to the mobile terminal uses a different frequency from the signal from the repeater.

2) The signal from the satellite to the mobile terminal uses the same frequency as that from the repeater.

Considering these two scenarios, two methods of combination are discussed: dual-band combination and single-band combination. The block diagram of two combination methods is shown in Fig. 2.

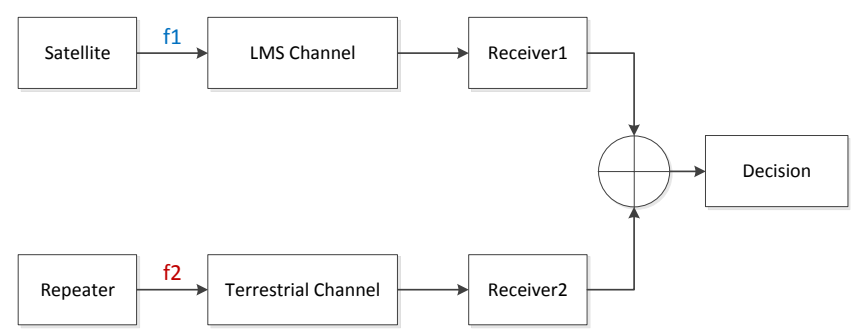

(a). Dual-band combination.

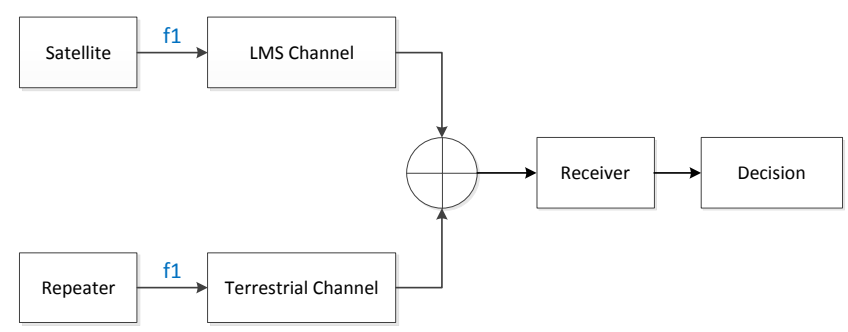

(b). Single-band combination.

Fig. 2. Methods for combining the signals.

In the first case, there are two receivers in the mobile terminal. The first one receives the signal in band Fig. 1 from 
satellite, and the other one receives the signal in band Fig. 2 from repeater. The two signals are combined after each receiver before the decision.

In the second case, there is only one receiver in the mobile terminal. It receives both the signal from the satellite and the signal from the repeater which are in the same band Fig. 1. These two signals are combined before the receiver.

\section{B. Models for Simulation}

Dynamic model can reproduce the probability density function of received signal power as well as dynamic behavior of fading and shadowing process. As a result, two dynamic models for simulation are made to check out the performance of each method:

1) Dual-band combination model

2) Single-band combination model
To illustrate modeling and simulation consideration, the model of dual-band combination is shown in Fig. 3.

A sequence of symbols $m(t)$ is first generated to transmit and a QPSK modulator is used in accordance with DVB-SH standard. Then the transmitting signal is separated into two paths: the upper path is the LMS channel in good state with Rician fading process, and the lower path is the terrestrial channel with Rayleigh fading.

The fading process is considered below. The received multipath signal is modeled as a Rayleigh process having Rayleigh distributed amplitude and uniformly distributed phase. This process is generated by complex addition of two independent stationary Gaussian processes. The spectral properties of the complex Rayleigh process are defined by an appropriate filter. As a compromise, a flat low-pass filter with a bandwidth of maximum Doppler frequency is used.

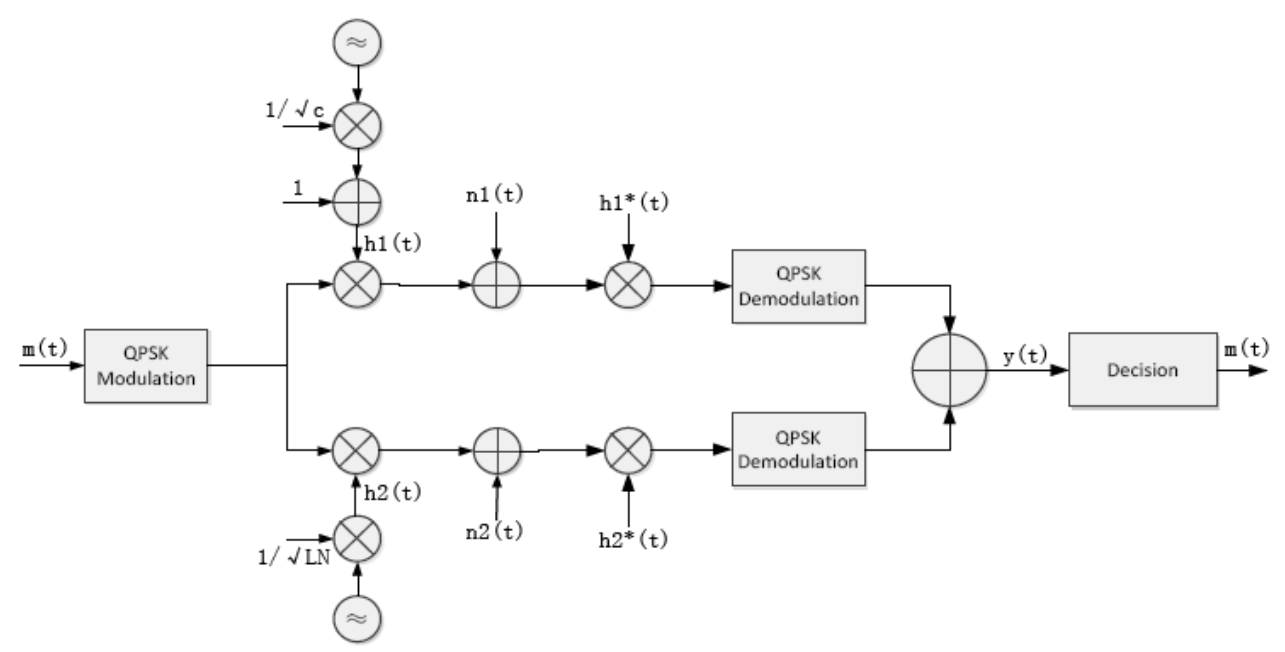

Fig. 3. Model of dual-band combination.

Based on the filtered Rayleigh process, the Rician fading is produced by attenuating the Rayleigh process power and adding a value of unity to represent the direct satellite signal component. The Rayleigh fading is generated by multiplying the Rayleigh process with a slow lognormal shadowing process. This approach has the advantage that very deep fades can be reproduced which is not possible when assuming constant multipath power. Situations with partly shadowed LOS are also included in the Rayleigh fading.

In order to approximate the dynamic behavior of the log-normal shadowing, the sort-term mean received signal power is chosen according to its probability density independently for each shadowing interval and kept constant during that interval.

After the implementation of each channel, an AWGN complex noise is generated. The noise is different for the good and the bad state because is different the power of signal for each state. For the good state the power of the signal depends on the Rician fading, while for the bad state depends on the Rayleigh fading.

\section{A. Simulated Results}

The complex envelope of received signal $y(t)$ of dual-band combination is obtained in Fig. 4.

The result shows that after combination of LMS good state channel and terrestrial channel, the mean power of the combined received signal remains the same with single LMS channel. To get detailed results, BER (Bit Error Rate) curve of dual-band combination is obtained in Fig. 5.

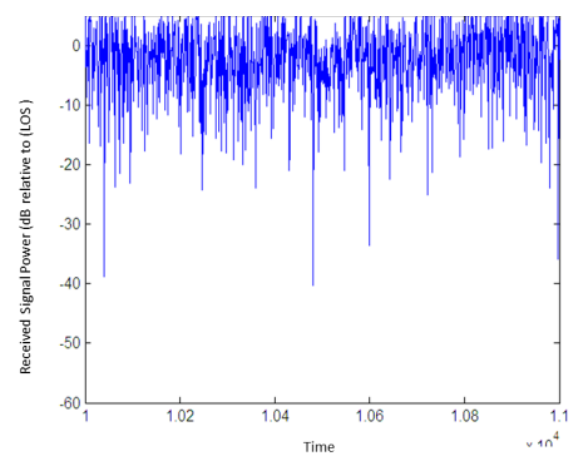

Fig. 4. Received signal of dual-band combination.

The yellow curve stands for the BER of received signal from terrestrial repeater. The blue curve stands for the BER of received signal from satellite. The dot black curve stands for the BER of dual-band combined signal. It can be seen that the performance of dual-band combination has a considerable improvement. The comparison of dual-band and single-band combination is shown in Fig. 6.

The red curve stands for the BER of single-band combined signal while the black curve stands for that of dual-band combined signal. 
The result shows that the dual-band combination has a better performance than single-band combination. The reason is that in single-band combination, the channel compensation is implemented after the combination of received signal which may leads to amplitude or phase counteraction. In dual-band combination, the channel compensation is considered for each receiver before combination, thus has a better performance. However, the mobile terminal is more complex for dual-band combination because it needs more receivers, also it needs more bandwidth.

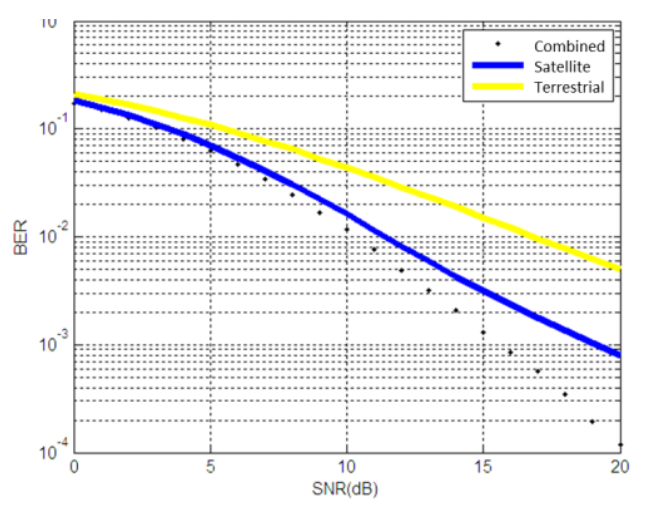

Fig. 5. BER of dual-band combined signal.

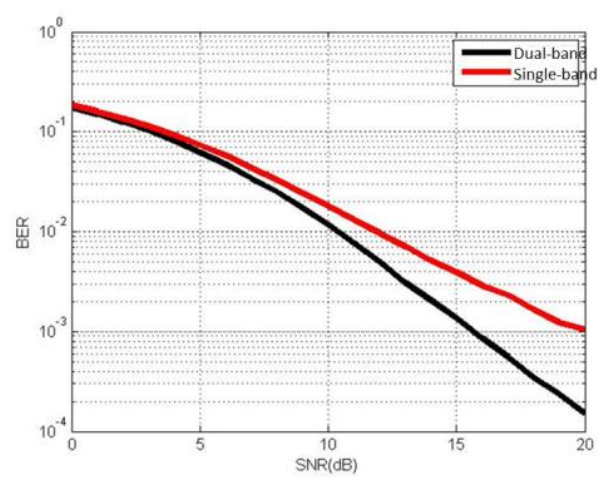

Fig. 6. Comparison of two methods.

\section{CONCLUSION}

In this paper, we provide and study method to improve the performance of DVB-SH mobile terminals. We make models for simulation based on dynamic behavior of different channels. The simulated result shows that both dual-band combination and single-band combination can improve the performance of mobile terminals, and there is a trade-off between the two methods.

For further study, we will focus on robust analysis of the methods. Different environment will be considered to check out whether the method is effective in different fading and multipath conditions. Terrestrial channel from repeater to mobile terminals will be re-modeled to take account of received signal power level and time delay.

\section{REFERENCES}

[1] DVB-SH standard (EN 302583 V1.1.1): "Framing structure, channel coding and modulation for Satellite Services to Handheld devices (SH) below 3 GHz," July 2007.

[2] F. P. Fontan, M. V. Castro, C. E. Cabado, J. P. Garcia, and E. Kubista "Statistical modelling of the LMS channel," IEEE Trans. On Vehicular Technology, vol. 50, no. 6, pp. 1549-1567, Nov 2001.

[3] E. Lutz, D. Cygan, M. Dippold, F. Dolainsky, and W. Papke, "The land mobile satellite communication channel-recording, statistics, and channel model," IEEE Trans. on Vehicular Technology, vol. 40, no. 2, pp. 375-386, May 1991.

[4] C. Loo and J. S. Butterworth, "Land mobile satellite channel measurements and modeling, Proceeding of the IEEE, vol. 86, no. 7, pp. 1442 -1463, July 1998.

[5] A. Jahn, "Propagation considerations and fading countermeasures for mobile multimedia services," International Journal of satellite Communications, vol. 19, no. 3, pp 223-250, May 2001.

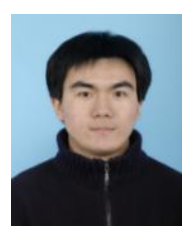

Zhou Hui received his master degree in computer applied technology from Beijing University of Posts and Telecommunications, China and master specialist degree in satellite communication systems from Institute Superior de l'Aeronautique et de l'Espace, France. He is currently working as an engineer at Institute of Telecommunication Satellite, CAST, Beijing, China. His research interests include satellite communication technology and systems. 